Rev. Elev. Méd. vét. Pays trop., 1979, 32 (2) : 123-129

\title{
Problèmes posés par la pathologie virale du mouton en zone sahélienne et soudano-sahélienne *
}

\author{
par P. BOURDIN (**)
}

\begin{abstract}
RÉSUMÉ
Les principales maladies virales du mouton actuellement connues sont au nombre de trois : la peste des petits ruminants, la clavelée ou variole ovine et l'écthyma contagieux.

La plus importante de ces affections est la peste des petits ruminants qui, par les complications bactériennes qu'elle entraine, décime régulièrement les jeunes animaux. Pour ces trois maladies, la prophylaxie se fait par l'utiljsation de vaccins à virus vivants.

A ce groupe on a rattaché les maladies dues aux Rickettsiales, les deux plus importantes étant chez le mouton, la heartwater et la rickettsiose générale ovine.

Enfin, sont décrites un certain nombre de maladies encore méconnues mais qui, à l'avenir, peuvent se révéler comme importantes: la blue tongue, les affections respiratoires à virus para-influenza III, les pneumoentérites à adénovirus et les affections digestives à rotavirus.
\end{abstract}

\section{INTRODUCTION}

Parler de problèmes dans cette communication, laisse entendre que dans le domaine de la pathologie virale du mouton les solutions sont loin d'être trouvées. En un sens ce terme est trop restrictif si l'on fait référence aux maladies virales actuelles les plus importantes pour l'élevage ovin de type extensif. En effet, les solutions existent et l'application en est faite depuis plusieurs années dans des unités pilotes. Mais, la généralisation, possible sur le plan technique, est freinée pour des motifs socio-économiques.

Les années de sécheresses antérieures, en modifiant les conditions de l'élevage bovin, ont valorisé la production des petits ruminants. L'espèce ovine longtemps ignorée par les programmes de recherche, sauf en pathologie, figure maintenant en bonne place dans les thèmes de la production animale. Les travaux et les applications qui en

(*) Communication présentée aux IX Journées médicales de Dakar, 15-20 janvier 1979.

(**) Chef du Service de Virologie. I. S. R. A., Laboratoire national de l'Elevage et de Recherches vétérinaires, B.P. 2057, Dakar, Sénégal. seront faites dans les unités pilotes amèneront la création de troupeaux ovins sélectionnés, à performances améliorées. Il est prévisible que ce nouveau type d'élevage, dit fermier par comparaison avec l'élevage actuel de parcours, entraînera l'éclosion de maladies infectieuses de deuxième génération où figureront en bonne place plusieurs viroses touchant surtout les jeunes.

Au cours de cette communication, nous rapporterons l'essentiel des connaissances acquises sur les viroses ovines actuelles. Nous évoquerons ensuite sous l'angle épidémiologique les principales affections virales à craindre dans le futur. La discussion qui terminera cet exposé nous permettra de dresser le bilan et d'en tirer les conséquences pour l'élevage ovin dans ces zones.

\section{LES PRINCIPALES MALADIES VIRALES RENCONTREEES ACTUELLEMENT CHEZ LES OVINS}

Indiquons que l'essentiel de nos connaissances concerne le Sénégal, mais les résultats 
peuvent être transposés à d'autres pays de l'Ouest africain situés sous la même latitude. Trois maladies virales sont répertoriées chez les ovins; par ordre d'importance on relève : la peste des petits ruminants (PPR), la clavelée ou variole ovine et l'écthyma contagieux. Nous évoquerons également les rickettsioses et les chlamydioses, affections qui, bien que n'étant pas dues à des virus, figurent dans cette communication.

\section{La peste des petits ruminants}

Elle domine de loin la pathologie ovine. Observée et décrite pour la première fois en 1940, en Côte-d'Ivoire (15), puis au Dahomey $(10)$; elle a surtout été étudiée au Sénégal $(7,8,5,6,18,22,23)$, au Nigéria $(21)$, au Tchad (28) et récemment aux Etats-Unis $(13,20)$.

Ne seront relatés ici que les points essentiels la concernant. Cette affection, propre aux petits ruminants, atteint chèvres et moutons avec cependant une nuance: chez les caprins on rencontre le plus souvent la forme aiguë ou "pestique ", tandis que chez les ovins on rencontre la forme subaiguè ou chronique à dominante pulmonaire dans laquelle l'étiologie virale primitive, véritable détonateur est relayée par des germes de sortie où dominent les bactéries du genre Pasteurella (d'où le nom de pasteurellose ovine que l'on trouve fréquemment dans les rapports).

Sur le plan épidémiologique, elle sévit cycliquement en zone d'élevage, pendant la saison humide, sous la forme de véritables épizooties décimant entre 40 et 60 p. 100 des individus sensibles, les jeunes et les adultes dépourvus d'immunité naturelle; puis l'épizootie s'arrête et 3 ou 4 ans plus tard ja population sensible étant redevenue suffisamment importante une nouvelle vague apparaît. La transhumance vers les régions agricoles pendant la saison fraîche favorise sa diffusion. La transmission se fait essentiellement par la voie nasale.

Le virus appartient à la famille des Paramyxoviridae. Cultivé sur cellule fotale de mouton en 1962 (18), son étude a été faite en 1967 et $1968(7,22)$. Virus à ARN monocatenaire, il possède une enveloppe et mesure entre 300 et $600 \mathrm{~nm}$. II se multiplie au niveau du cytoplasme et sort de la cellule par bourgeonnement de la membrane cytoplasmique. Il est fragile, détruit par la chaleur et l'éther ; il résiste peu aux variations de $\mathrm{pH}$. Sur le plan antigénique il est étroitement lié au virus de la peste bovine (P. B.) : la fixation du complément ne permet pas de les distinguer; seule la séroneutralisation croisée donne une différence dans les titrages (20).

Inoculé aux bovins par voie parentérale, il se multiplie et donne lieu à une courte excrétion nasale, sans signe clinique. Ces bovins acquièrent une solide immunité contre la P. B. Réciproquement, l'inoculation aux moutons d'un virus $\mathrm{P}$. B. atténué par passage en série sur lapin ou par cultures cellulaires les rend résistants vis-à-vis du virus PPR.

Sur le plan clinique, la maladie se traduit par une hyperthermie plus ou moins prononcée avec aspect frileux de l'animal; on note, en outre, jetage, larmoiement et éternuements. Le son de la voix est modifié, suite à l'inflammation des amygdales. A ce stade apparaissent les complications pulmonaires plus ou moins graves chez les ovins et les complications digestives avec diarrhée et déshydratation. Soit la mort survient entre 5 et 8 jours, ou soit on passe au stade chronique avec une dominante respiratoire (bronchopneumonie et pleuropneumonie). Il est facile d'isoler à ce stade des pasteurelles dans le liquide pleural ou le liquide péricardique. L'isolement de mycoplasmes est beaucoup plus rare.

Sur le plan diagnostic, les signes cliniques et nécropsiques, accompagnés du contexte épidémiologique sont suffisants. Au niveau du laboratoire la confirmation est donnée par l'isolement du virus ; le test de précipitation en milieu gélifié n'est pas spécifique. L'isolement est délicat et ne peut être réussi qu'à partir des ganglions lymphatiques, ou des lésions pulmonaires prélevées sur un animal en pleine hyperthermie et au début de la maladie. L'isolement dans le sang et le mucus nasal peut être réalisé à condition d'intervenir dans les premières $72 \mathrm{~h}$. Deux tests mériteraient d'être mis au point pour le diagnostic de la PPR : l'immunofluorescence d'une part et l'immuno-enzymologie d'autre part.

Dans le domaine de la prophylaxie, la protection est obtenue par l'injection du virus de la P. B. modifié par passages sur cellule rénale de fœtus de veau ; c'est ce virus modifié qui est utilisé pour la prophylaxie de la P. B. Le vaccin obtenu est constitué par la souche RP KO/BK 65 passages (27). L'utilisation d'un virus hétérologue pour la protection des petits ruminants remonte à 1956, où déjà on vaccinait les caprins avec le virus P. B. lapinisé. En 1969, le vaccin « Tissupest " est testé au Dahomey (Bénin) et 
au Sénégal. A partir de 1970 il est utilisé chaque année dans le village de Thionar près de Thiès sur 800 petits ruminants. Enfin depuis 1976, 5000 petits ruminants vivant dans les Unités expérimentales de l'IRAT, à Koungheul et Koumbidia le reçoivent régulièrement. $A$ Thiès et dans les unités de l'IRAT le vaccin est injecté une fois par an à tous les animaux âgés de plus de 2 mois. La vaccination contre la pasteurellose n'est pas associée. Les contrôles sérologiques, faits avant et après vaccination sur un échantillonnage de 100 ovins, par recherche des anticorps neutralisants, donnent les résultants suivants :

Taux de protection avant et après vaccination.

\begin{tabular}{|l|l|l|}
\hline \multicolumn{1}{|c|}{ Avant vaccination } & $\begin{array}{c}5 \text { mois après } \\
\text { vaccination }\end{array}$ \\
\hline $\begin{array}{l}\text { Age : moins de } \\
6 \text { mois }\end{array}$ & $\begin{array}{l}\text { Age : plus de } 6 \\
\text { mois }\end{array}$ & Age non spécifié \\
\hline 10,5 p. 100 & 21,7 p. 100 & 94 p. 100 \\
\hline
\end{tabular}

L'âge de la primo-vaccination est fixé à 2 mois car les jeunes agneaux acquièrent leur immunité après la naissance par l'ingestion du colostrum puis du lait. Or les immunoglobulines du colostrum sont transférées à la circulation sanguine du jeune par passage à travers la paroi intestinale. Ce passage cesse dès les premières $24 \mathrm{~h}$ avec l'imperméabilisation de la muqueuse intestinale. L'apport d'immunoglobulines à la cavité digestive se poursuit pendant toute la lactation mais il s'agit alors d'une immunité passive locale. Entre la $3^{\text {e }}$ semaine et le $2^{e}$ mois après la naissance, le taux d'anticorps sériques fléchit rapidement et le jeune agneau va rapidement se trouver sans protection d'autant que la porte d'entrée habituelle du virus située au niveau des voies aériennes supérieures est démunie de protection locale passive.

\section{La clavelée ou variole ovine}

Signalée en 1968 dans la région de Tambacounda (16), puis en 1970 à Kaolack et à Dakar, elle se rencontre actuellement dans toutes les régions. Son extension est facilitée lors de la commercialisation des ovins avant la fête de la Tabaski.

La maladie ne touche pas les caprins contrairement à ce qui est observé au Kenya (14). L'aspect des lésions cutanées est différent de celui décrit pour la clavelée sévissant sur le pourtour de la Méditerranée.
Après une phase d'hyperthermie accompagnée d'anorexie, on remarque l'apparition 'de nodules sous-cutanés localisés à la tête et à la partie antérieure du corps. La formation de vésicules et de pustules est plus rare; elle se rencontre chez les femelles au niveau de la mamelle ou chez les jeunes sur la muqueuse buccale. L'évolution peut durer entre 2 et 4 semaines; les animaux subissent une importante perte de poids. On peut observer quelques formes graves à localisations pulmonaires, en général mortelles; graves sont aussi les formes digestives des jeunes.

Dans les effectifs atteints, le pourcentage des malades varie entre 25 et 50 p. 100 . En général, l'évolution va vers la guérison sauf dans les formes graves. Le préjudice pour l'éleveur est sensible, d'autant que les malades, éliminés du circuit commercial, subissent une perte de poids non négligeable. La transmission se fait par contact, les insectes hématophages peuvent également intervenir.

Le virus de la clavelée appartient à la famille des Poxciridae, sous groupe III (dans lequel figurent les virus de la variole caprine et de la lumpy skin disease. Virus à $\mathrm{ADN}$, il mesure entre 200 et $300 \mathrm{~nm}$ et a la forme d'une brique. Le virus, isolé en 1970 à Dahra, est cultivé sur cellules rénales de fœtus de mouton. Après 30 passages, il a perdu son pouvoir pathogène et ne provoque qu'une pustule après inoculation par scarification à la face interne de la cuisse.

La prophylaxie est réalisée avec la souche $\mathrm{RM} / 65$, atténuée par passage sur cellule rénale de mouton (29). Le vaccin est injecté par la voie sous-cutanée, l'immunité est totale 15 jours après, sa durée est supérieure à une année (29).

Sur le plan immunologique, il reste à établir les relations du virus claveleux isolé au Sénégal avec ceux de la variole caprine et de la lumpy skin disease.

\section{L'ecthyma contagieux ou dermite pustu- leuse de la chèvre et du mouton (12)}

La maladie est connue des éleveurs de moutons. Elle touche les agneaux, mais peut se rencontrer chez les adultes. Elle se caractérise par la formation de vésico-pustules au niveau de la commissure des lèvres qui, rapidement, se transforment en papules croûteuses. On observe parfois l'extension aux lèvres supérieures et inférieures. Les rares complications sont dues à l'envahissement des lésions par le bacille de la nécrose, Spherosphorus necrophorus. En général, 
l'ecthyma évolue vers la guérison en 2 à 4 semaines. Le préjudice est d'ordre économique ; les animaux perdent du poids, suite à l'impossibilité de se nourrir. Le virus appartient également aux Poxviridae. Cette dermatose peut devenir un obstacle à l'élevage intensif des jeunes pour l'engraissement. La prophylaxie médicale en est simple : un vaccin peut être préparé à partir de croûtes virulentes desséchées et broyées en glycérine. La vaccination se faisant par scarification à la face interne de la cuisse.

\section{Les maladies dues aux Rickettsiales}

Les Rickettsiales sont des micro-organismes petits, souvent pléomorphes qui s'observent sous l'aspect d'éléments ou de corps élémentaires et sont habituellement intra-cellulaires. Elles peuvent donner lieu à des « corps initiaux », à des inclusions. Leur composition chimique $\mathrm{ADN}$ et ARN, leurs enzymes et leur paroi, les rapprochent des bactéries; cependant, ces germes ne prennent pas la coloration de Gram et se cultivent comme les virus, sur cellules vivantes, cufs embryonnés, cultures de tissus et animaux (9).

Sur un plan taxonomique, rappelons que les Rickettsiales se divisent en 3 familles :

- Les Rickettsiaceae, germes responsables de maladie chez les vertébrés, généralement transmis par des arthropodes.

- Les Chlamydiaceae, parasites intracellulaires des vertébrés, non transmis par les arthropodes.

- Les Bartonellaceae et Anaplasmataceae que l'on trouve dans ou sur les érythrocytes.

Dans le cadre de cet exposé, nous nous limiterons aux deux premières familles, la $3^{\text {e }}$ étant traitée dans le cadre des hémo-parasites.

Les Rickettsiaceae se divisent encore en 3 tribus :

- Les Rickettsiae pathogènes pour les vertébrés dont l'homme;

- Les Erhlichiae pathogènes uniquement pour les animaux;

- Les Wolbachiae trouvées uniquement chez les arthropodes.

Nos études sont limitées aux maladies dues aux Erhlichiae et Chlamydiaceae. Cette digression taxonomique nous a paru indispensable.

a) Les maladies des ovins dues aux Ehrlichiae

Deux germes sont trouvés chez les ovins Cowdria ruminantium, parasite des cellules endo- theliales et responsable de la heartwater et Erhlichia ovis, parasite des globules blancs, responsable de la rickettsiose générale ovine.

\section{- La Heartwater}

C'est une maladie virulente inoculable, non contagieuse, particulière aux ruminants et transmise par les tiques du genre Amblyomma (26). Reconnue en 1858 en Afrique du Sud, on la trouve dans toute l'Afrique intertropicale et Madagascar, partout où se trouve Amblyomma variegatum. Elle touche les ruminants sauvages et domestiques. Le mouton constitue l'espèce la plus sensible, surtout les animaux importés ou en état de moindre résistance. Chez les ovins, on rencontre la forme suraiguë, mortelle en quelques heures. L'animal apparemment en bonne santé, tombe brusquement sur le sol, pédale, se débat et meurt. La forme aiguë dure 2 à 5 jours, avec hyperthermie, essoufflement, dyspnée, assourdissement des bruits cardiaques, crises convulsives, tournis, décubitus et mort. Enfin la forme frustre, fréquente chez les animaux locaux naturellement prémunis.

Le diagnostic se fait par l'examen nécropsique. On note, hydropéricarde, splénomégalie, gastroentérite. Mais surtout par la recherche immédiate des rickettsies au niveau des cellules endothéliales, soit à partir de frottis faits avec le produit de grattage des grosses artères, soit par écrasement de fragments de cortex cérébral. Il est essentiel de fixer les frottis sur le champ, à l'alcool méthylique. La coloration au Giemsa pourra ainsi être faite plus tard. Cowdria ruminantium n'est ni cultivable sur cellules, ni inoculable aux animaux de laboratoire. Elle se conserve à $-70^{\circ} \mathrm{C}$ ou dans l'azote liquide et s'entretient par passages sur moutons mérinos. Il n'existe pas de diagnostic sérologique. La prophylaxie médicale est irréalisable, la seule méthode préventive est la destruction des tiques par bains ou douches ixodicides hebdomadaires. Le traitement n'est efficace que s'il est pratiqué avant l'apparition des troubles nerveux et cardiaques. On fait une injection par voie endoveineuse de chlortétracycline (Auréomycine) à la dose de $5 \mathrm{mg} / \mathrm{kg}$ à reprendre 2 à 3 jours de suite. L'oxytétracycline (Terramycine) est également efficace. Dans les foyers de maladie naturelle, il est conseillé de traiter l'ensemble des animaux par une distribution orale de Terramycine à la dose de $400 \mathrm{mg}$ par jour pendant 4 jours (eau de boisson). 


\section{- L'erhlichiose générale ovine}

Maladie due à Erhlichia ovis, transmise par les tiques vivant en zone sahélienne, Hyalomma et Boophilus, elle a été décrite pour la première fois en 1941 (11). Elle peut également se transmettre par les Amblyomma en zone humide. Le plus souvent l'erhlichiose générale ovine sévit à l'état latent ou fruste, les animaux étant prémunis dès leur plus jeune âge et se réinfectant périodiquement (30). Cet équilibre peut être rompu lors de disettes, de modification des conditions météorologiques diminuant la prolifération des tiques donc la prémunition naturelle, et par les maladies infectieuses ou parasitaires intercurrentes.

Sur le plan clinique, elle se traduit par une température élevée au début, du jetage, un amaigrissement plus ou moins prononcé, des signes d'anémie, l'arrêt de la rumination, la constipation, quelques signes, nerveux parfors sont associés. L'animal reste couché, " la tête regardant le flanc ", il meurt en une semaine. A l'autopsie, on note un hydropéricarde léger associé à un hydrothorax et un hydropéritoine. Le diagnostic est confirmé par la mise en évidence d'Erhlichia ovina dans les monocytes et également dans les polynucléaires sur frottis sanguins colorés au May-Grunvald-Giemsa. La prophylaxie et le traitement se réalisent dans les mêmes conditions que pour la heartwater. Une remarque : on a réussi à cultiver Erhlichia canis sur culture de monocytes (25) de chien. On peut espérer réussir le même type de culture à partir des monocytes de mouton et obtenir ainsi une souche d'antigène à diverses fins.

- La rickettsiose conjonctivale du mouton

Décrite en 1945 (30) en Afrique intertropicale, elle se caractérise par une conjonctivite (uni) ou bilatérale, et peut se compliquer par une kératite. L'évolution dure de 4 à 10 jours et se termine en général par la guérison sauf complications. Le traitement est à base de collyre et surtout de pommade contenant de la chlortétracycline ou de l'oxytétracycline à 4 p. 100.

b) La chlamydiose (Chlamydia psittaci ovis) :

Ces deux dernières années, des enquêtes sérologiques ont été poursuivies sur des animaux tout-venant (unités expérimentales). L'antigène Roger BELLON a été utilisé dans une microréaction de fixation du complément. Des titres faiblement positifs ont été notés, sans qu'il soit possible de conclure à l'existence de la maladie (réactions croisées avec d'autres affections non exclues). La mise en évidence de la chlamydiose reste subordonnée à l'observation de cas d'avortements infectieux dans des foyers déterminés signalés au Laboratoire. Seuls des titres en anticorps élevés ou mieux, l'isolement de souches de Chlamydia à partir d'avortons sur cufs ou cultures cellulaires, peuvent permettre de conclure à l'existence de l'affection chlamydienne et à une incidence économique.

Il en va de même de la fièvre Q (Coxiella burneti).

\section{LES MALADIES DITES D'AVENIR}

On a regroupé sous ce titre des affections dont l'existence est prouvée mais qui pour l'instant n'ont pas encore d'incidence sur le plan sanitaire et des maladies virales inconnues ou peutêtre méconnues, mais qui peuvent se révéler présentes après des enquêtes épidémiologiques approfondies. Dans tous les cas, ces affections méritent de retenir l'attention des pathologistes car, leur incidence peut suivre les progrès réalisés sur les plans zootechnique et nutritionnel.

\section{La bluetongue}

La bluetongue figure parmi les arboviroses. Elle est transmise par diverses espèces de Culicoides dont la prolifération est favorisée par l'humidité, les pluies abondantes et la température. Le virus de la bluetongue appartient à la famille des Reoviridae et comprend à ce jour 16 types sérologiques différents. Parmi les hôtes habituels du virus, on relève le mouton, la chèvre, les bovins, le chameau et diverses espèces d'antilopes. La maladie est connue en Afrique du Sud depuis 1876. En Afrique intertropicale, le premier cas signalé remonte en 1925 , sur des moutons importés (12). Depuis, il est prouvé par des enquêtes sérologiques $(19,34)$ et isolements du virus $(3,4)$ que la maladie existe à l'état endémique. Le virus de la bluetongue persiste dans la nature selon un cycle où interviennent : les culicoïdes, les bovins, les antilopes et peut-être certains rongeurs. Les moutons et surtout les animaux importés jouent le rôle de révélateur.

Sur le plan clinique, la maladie se caractérise par un syndrome fébrile avec une congestion violente de la muqueuse buccale, puis apparait une stomatite ulcéro-nécrotique avec œdème de la langue et des lèvres, jetage séro-sanguinolant et gastro-entérite hémorragique. La morbidité dépasse 70 p. 100 et la mortalité atteint 
50 p. 100 . La prophylaxie est réalisable par l'emploi de vaccins à virus atténués. Leur utilisation reste assez complexe en raison des différences antigéniques entre les divers types. L'utilisation doit en être réservée aux animaux importés (34). Au Sénégal, on considère la bluetongue comme une maladie d'avenir et dans un premier temps, il convient de faire une enquête sérologique pour évaluer son incidence.

\section{Les affections virales de l'appareil digestif et respiratoire des agneaux (1)}

11 s'agit là d'un groupe de maladies, de découverte récente, que l'on rencontre dans les grands centres d'engraissement où sont mélangés des animaux de diverse provenance. Le stress, causé par la promiscuité et l'alimentation artificielle, fragilisent les agneaux à l'égard de virus qui, normalement, ont une virulence faible. A cela s'ajoutent les complications bactériennes sur un terrain en état de moindre résistance. Si l'action des virus se traduit surtout par une perte de poids, l'intervention des bactéries aggrave le pronostic. Ce type de maladies est encore inconnu en Afrique intertropicale mais l'évolution qui se dessine dans l'élevage des ovins doit les faire craindre, d'où l'intérêt de les étudier maintenant.

\section{a) Les affections respiratoires dues au virus parainfluenza (3)}

Les premiers isolements de virus PI 3 à partir du mouton remontent en 1966, en GrandeBretagne. Depuis, le virus a été retrouvé dans les produits pathologiques d'agneaux atteints de maladies respiratoires dans divers pays, que ce soit lors d'une épidémie naturelle ou après une transmission expérimentale. La mise en évidence est réalisée par l'inoculation du mucus nasal à des cultures de cellules foetales de rein d'agneau. Le sérum des animaux convalescents possède des anticorps inhibant l'hémagglutination à un titre important. Au Sénégal, la maladie n'existe pas à l'état clinique, mais une enquête sérologique (2) faite à partir d'échantillons provenant de moutons vivant dans diverses régions, révèle que 25 à 50 p. 100 des ovins adultes possèdent des anticorps inhibant l'hemagglutination à la dilution $1 / 50$. Le contact avec le virus existe mais la maladie évoluant sous une forme bénigne, il n'a pas été possible d'isoler le virus jusqu'ici.

\section{b) Les pneumoentérites à adénovirus}

Maladies aiguës des jeunes, les pneumoentérites à adénovirus se rencontrent surtout dans les unités d'élevage intensif lors de l'introduction de nouveaux animaux. Une semaine après leur arrivée, ils sont atteints de diarrhée avec hyperthermie puis dans les 2 à 3 jours, apparaissent des troubles respiratoires : jetage, éternuement et conjonctivite. Quelques morts rapides peuvent survenir. L'autopsie révèle au niveau du poumon, des lésions d'atelectasie et de bronchite aiguë avec prolifération lymphohistiocytaire dans les cloisons inter-alvéolaires et hyperplasie des follicules lymphatiques péribronchiques. $\mathrm{Si}$ les conditions d'hygiène sont déplorables : entassement, mauvaise ventilation, humidité excessive, la forme aiguë se transforme rapidement en forme chronique, suite à l'intervention de Mycoplasma ovipneumoniae, Pasteurella hemolytica ou de corynebactéries SPP.

Jusqu'à présent, les adénovirus du mouton sont classés en 5 types sérologiques; récemment on a trouvé chez un agneau un type 2 bovin. Au Nigeria, on vient d'isoler 2 types d'adenovirus chez des caprins associés au virus PPR (17). Après typage, les auteurs signalent que ces 2 adenovirus sont différents de ceux jusqu'ici connus chez les ovins. Une enquête sérologique sommaire, faite sur des moutons vivant dans la même zone, montre que ceux-ci possèdent des anticorps contre les souches d'adenovirus mises en évidence récemment.

\section{c) Les affections digestives à rotavirus}

La découverte de ces virus est récente : ils sont isolés lors de gastroentérites aiguës du porcelet, du veau et de l'agneau. Au début, ces virus décrits comme des Reoviridae, sont maintenant considérés comme un sous-groupe des Reoviridae et dénommés rotavirus (24). Expérimentalement, on a pu reproduire leur action pathogène chez des agneaux gnotobiotiques (33). Une enquête faite en Ecosse (32), révèle que dans plusieurs troupeaux, 38 p. 100 des adultes et 56 p. 100 des agneaux ont eu un contact avec des rotavirus. En Afrique sahélienne, aucune recherche n'a été entreprise mais il convient de s'y intéresser au même titre que les adénovirus et le virus parainfluenza III.

\section{DISCUSSION ET CONCLUSION}

L'élevage des petits ruminants et des moutons en particulier, n'a pas connu sur le plan sanitaire les mêmes attentions que l'élevage bovin. Or, depuis moins d'une décennie, ce type d'élevage 
mieux adapté au milieu sahélien et aux conditions climatiques actuelles a été valorisé. Toutefois, les mesures de prophylaxie médicale sont encore très limitées sur le terrain, malgré les démonstrations faites par le Laboratoire. La peste des petits ruminants en est le plus bel exemple, du moins au Sénégal. En effet, cette virose et les complications qui en résultent, la font considérer comme la maladie la plus meurtrière pour les ovins. Revenant tous les 4 ou 5 ans, elle décime dans les troupeaux les sujets les plus sensibles, en général les jeunes. On peut dire, en exagérant peut-être, que la PPR et ses complications constituent le processus naturel le plus efficace pour limiter la prolifération des petits ruminants. N'oublions pas cependant que si les virus jouent parfois le rôle initiateur, d'autres agents pathogènes interviennent chez le mouton, sans oublier les accidents nutritionnels et les intoxications : en particulier, les intoxications végétales dont nous avons déjà observé 3 cas chez les moutons pendant la saison sèche.

\section{SUMMARY}

\section{Sheep viraI diseases in the Soudano-Sahelian area}

The three main viral diseases of sheep are: peste des petus ruminants $(P . P . R$.), sheep pox and contagious ecthyma.

The most important affection is the peste des petits ruminants which is usually fatal for young animals because of bacterial complications.

The prevention of these three viral diseases is practiced by live virus vaccines.

This group of diseases includes also rickettsial infections, the most deadly ones for sheep being the heartwater and Rickettsia ovina.

Lastly the author describes some diseases which are still misunderstood butcan become worth of interest in the future : blue tongue, viral para-influenza III, adenoviral pneumoenteritis, rotaviral digestive affections.

\section{RESUMEN}

\section{Problemas planteados por la patología viral del ganado lanar en zona} saheliana y sudano-saheliana

Las tres principales enfermedades virales del ganado lanar, actualmente conocidas, son las siguientes : la peste de los pequeños rumiantes, la viruela ovina y el ectima contagioso.

La más importante es la peste de los pequeños rumiantes que, por las complicaciones bacterianas que ocasiona, diezma regularmente a las crias. La profilaxia de dichas enfermedades se hace por la utılización de vacunas a virus vivos.

Con este grupo se relaciona las enfermedades causadas por las Rickettsiales, las dos más importantes siendo la heartwater y la rickettsiosis general ovina.

Por fin, se describen un cierto número de enfermedades todavia desconocidas pero que en lo sucesivo pueden hacerse importantes: la lengua azul, las afecciones respiratorias a virus para-1nfluenza III, las pneumoenteritis a adenovirus y las afecciones digestivas a rotavirus

\section{BIBLIOGRAPHIE}

5. BOURDIN (P.). La peste des petits ruminants et sa prophylaxie au Sénégal et en Afrique de ]'Ouest. Rev. Elev. Méd. vét. Pays trop., 1973, 26 (4) : 71-74

6. BOURDIN (P.) et DOUTRE (M.). La peste des petits ruminants au Sénégal. Données nouvelles. Rer. Elev. Méd. vét. Pays trop., 1976, 29 (3) . 199-204.

8. BOURDIN (P.). LAURENT (A.). Etat actuel des recherches sur la prophylaxie médicale de la peste des petits ruminants. XL Session générale de I'O. I. E. Paris, ma1 1972. Rapport $\mathrm{n}^{\circ} 2$

9. CAPPONI (M.). Diagnostic des rickettsiales au laboratoire. Paris, Maloine, 1974.

13. DARDIRI (A. H.), DE BOER (C. J.) et HAMDY (F M.). Response of american goats and cattle to peste des petits ruminants virus. Proc. 19th a Mtg. Am. Ass vet. Lab. Diag, 1977 : 337-344.

(*) La bibliographie complète (34 réf.) sera communiquée aux personnes qui en feront la demande auprès de la Rédaction de la Revue.
15. GARGADENNEC (L.), LALANNE (A.). La peste des petits rumınants. Bull. Serv. zoot. A. O. F., 1942, 5: 16 .

17. GIBSS (E. P. J.), TAYLOR (W. P.), LAWMAN (M. J. P.). The isolation of adenoviruses from goats affected with peste des petits ruminants in Nigera. Res. vet. Sci., 1977, 23 (3) : 331-335.

23. MORNET (P.), ORUE (J.), GILBERT (Y.), THIERY (G.), SOW (M.). La peste des petits ruminants en Afrique occidentale françaıse et ses rapports avec la peste bovine. Rev. Elev. Méd. vét. Pays trop., 1956, 9 (4) : 313-342.

27. PLOWRIGHT (W.), FERRIS (R. D.). Studies with rinderpest virus in tissue culture. The use of attenuated culture virus as a vaccine for cattle. Rés. vet. Sci., 1962, 8 . 172-182.

28. PROVOST (A.), MAURICE (Y.), BORREDON (C.). La peste des petıts ruminants existe-t-elle en Afrique centrale? XL Session générale du Comité de l'O. I.E., Paris, mai 1972. Rapport 202. 